\title{
Patient engagement or information overload: patient and physician views on sharing the medical record in the acute setting
}

\author{
Authors: Zoë Fritz, ${ }^{A}$ Alex Schlindwein ${ }^{B}$ and Anne-Marie Slowther ${ }^{C}$
}

Background: Patient and professional views about the impact of providing full real-time access to the medical record in the in-hospital setting are unknown.

Methods: Likert-scale and free-text validated questionnaire survey of physicians and patients from acute medical units in two hospitals. The questionnaire explored recent experiences; views on the formation of trust, and views on sharing either the entire medical record or a summary.

Results: Two-hundred and forty-eight patient questionnaires (62\% response rate) and 32 physician questionnaires $(21 \%$ response rate) were returned. Twenty-seven per cent of patients did not recall being told their diagnosis. Doctors and patients differed on what practices that they believed built trust.

Eighty-one per cent of patients supported the idea of having access to the full medical record (for empowerment; the right to information about oneself; as an aide-memoire for discussion). Doctors feared it might provoke anxiety and change the nature of what was written. A written lay summary record was preferred by doctors and patients.

Conclusions: The current system of providing information verbally to patients is inadequate. Patients want more information and are less concerned than physicians about potential negative effects of real-time access to their records. Patient access to medical records (in both full and summary forms) should be evaluated.

KEYWORDS: Access to information, medical records, ethics, autonomy, disclosure of information

\section{Introduction}

Medical documentation in outpatients has changed; patients are now routinely copied into letters, which are often written in lay terms, and there are many initiatives to give patients access

Authors: ${ }^{\mathrm{A}}$ consultant in acute medicine, Wellcome fellow in society and ethics, The Healthcare Improvement Studies (THIS) Institute, Cambridge, UK and Warwick Medical School, Coventry, UK;

${ }^{B}$ medical student, University of Cambridge, Cambridge, UK; $C_{\text {reader }}$ in ethics, Warwick Medical School, Coventry, UK to entire outpatient records. ${ }^{1}$ Patient-held records are common for specific diseases ${ }^{2}$ and maternity services; ${ }^{3}$ patient access to primary care records is growing. ${ }^{4}$ Proponents for improving patients' access to their medical records argue that this increases transparency, patient autonomy and engagement. ${ }^{5-7}$ Studies investigating the impact of sharing records in outpatient settings have found that doing so can increase patient empowerment, ${ }^{1}$ improve safety ${ }^{8}$ and, despite physician fears, does not increase the number of patient requested visits. ${ }^{9}$ Similar findings were revealed in a systematic review of the use of patient portals (electronic access to parts or all of the medical record). ${ }^{10-13}$

In the UK, patients rarely look at their inpatient medical record, despite their legal right to do so. ${ }^{14}$ We did not identify any published studies evaluating the impact of providing full real-time access to the medical record in the inpatient setting, and only one providing a daily written summary record. ${ }^{15}$

Given the UK government's emphasis on patient access to all of their record, and the lack of evidence of its impact in an inpatient setting, we aimed to explore the attitudes and views of patients and physicians with regard to sharing patient records. We chose the acute medical setting because here patients need to entrust their care to physicians they have never met before, for conditions, treatments and investigations that are often unfamiliar. In this context, offering patients full, real-time access to the medical record might have more significant implications, both positive and negative.

\section{Methods \\ Questionnaire design and validation}

We carried out a questionnaire survey of acute medicine physicians, and patients who had been admitted to an acute medical unit. The questionnaire development was informed by themes identified in interviews with 12 patients and 13 acute medicine physicians conducted as part of a mixed-methods project.

Closed Likert-scale score (1-5) and open questions requiring freetext responses were included. Physician and patient questionnaires had similar questions. Wording was modified following feedback from the Cambridge University Hospitals Patient and Public Involvement panel.

Face validity was assessed using a 'thinking aloud'16 exercise with three lay and four physician volunteers; questionnaires were revised and retested. See Supplement S1 for questionnaires. 
The questionnaire explored:

> recent experience of being an inpatient (patient participants) or frequency of certain practices (physician participants)

$>$ perceptions of the impact of sharing all or part of the medical record in the acute care setting, in real-time

$>$ factors influencing patient's trust in their physician - in particular relating to information sharing.

Recruitment, eligibility and consent

Physicians were recruited from delegates at the Society of Acute Medicine conference held in Birmingham in 2017; paper copies of the questionnaire with stamped addressed envelopes were distributed at the conference directly to delegates by Zoë Fritz (ZF). An email reminder was sent 10 days later.

Patient participants were recruited from two sites: a large teaching hospital using electronic medical records and a smaller hospital serving a diverse ethnic population that used hand-written patient records. Current practice at both hospitals was to give verbal information to patients, and a written summary on discharge (to the patient and general practitioner). No patient portals were available for use. Patients over the age of 18 who were admitted acutely with a medical problem to one of the study sites were eligible. Patients were excluded if they lacked capacity to consent or did not speak English. Patients were identified by a research nurse when they were ready for hospital discharge (by speaking with the nursing staff on the ward) and given the questionnaire with a stamped addressed envelope. If a patient had difficulty reading or completing the form, the research nurse helped them to do so.

Completion of the questionnaire was taken as consent to participate in the survey.

\section{Ethics approvals}

Approvals were obtained from East of England Cambridgeshire and Hertfordshire Regional Ethics Committee, the Health Research Authority, and Research and Development Departments of participating institutions.

\section{Data analysis}

Questionnaire data were inputted into an MS Excel database. Quantitative data were analysed using descriptive statistics. A median ( $\mathrm{m}$ ) was calculated as the measure of central tendency for the non-parametric Likert data. This is presented (as a score of $1-5$, with 1 being the most negative and 5 being the most positive) to better demonstrate the heterogeneity of responses than a simple positive/negative split. ${ }^{17}$ Percentage responses to some questions are also provided for ease of interpretation.

Thematic analysis of the free text answers was carried out by ZF and Alex Schlindwein (AS).

\section{Results}

\section{Response rate}

Physician questionnaire response rate was 21\% (32/150). Patient response rate was $81 \%$ (87/128 in site A and 161/179 in site B). Age ranged from 19-91 for patient respondents, with most in the 61-75 bracket; year of qualification ranged from 1970-2015 for the physician respondents, with the largest proportion having qualified between 2000-9 (see Supplement S2 for full demographic data).

\section{Patient experiences of their recent admission with an acute illness}

Patients reported receiving a variety of information (see Supplement S3), but $27 \%$ did not remember being told their likely diagnosis. Only $61 \%$ reported being told what treatments they were given.

While most $(68 \%)$ patients felt they could almost always trust their physician $(m=4.77)$, only $53 \%$ 'almost always' felt they could ask questions $(m=4.56)$, and fewer $(32 \%)$ reported actually doing so $(m=3.75)$. Very few patients felt they were given too much information, but there was variability in whether more information was desired (Fig 1).

\section{Sharing full or summary medical records}

Most patients (81\%) agreed or strongly agreed that the medical record should be shared $(m=4.28)$, in contrast with $41 \%$ of physicians $(\mathrm{m}=3.09)$.

The majority of both patients and physicians thought sharing medical records would increase trust (patients $m=4.24$; physicians $m=3.65$ ), and facilitate or generate questions (patients $m=4.24$; physicians $m=4.26$; see Fig 2). While patients thought that having access to the record would increase certainty $(m=4.19)$ physicians
Did you understand what you were being told? Were there times when you wanted more information? Were there times when you were given too much information? Did you feel able to ask questions? Did you ask questions? If so, did you feel that they were answered? Did you feel that you could trust your doctors?

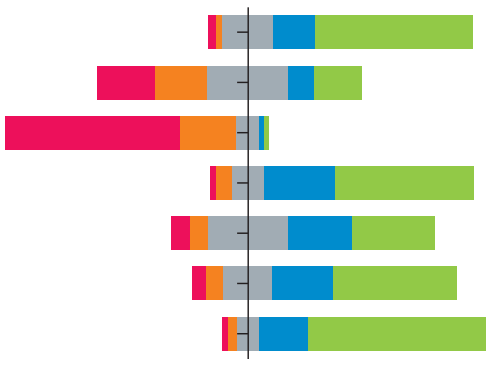

Percentage of responses Never Rarely Sometimes Often Almost always Scale (for reference) $\begin{array}{lcccc} & \mid & \mid & \mid & \mid\end{array}$
Fig 1. Patient responses from part 1 of the questionnaire. The fivepoint Likert data are plotted as a diverging stacked bar graph; each bar equates to $100 \%$ of the response to that question, centred about the neutral responses. 


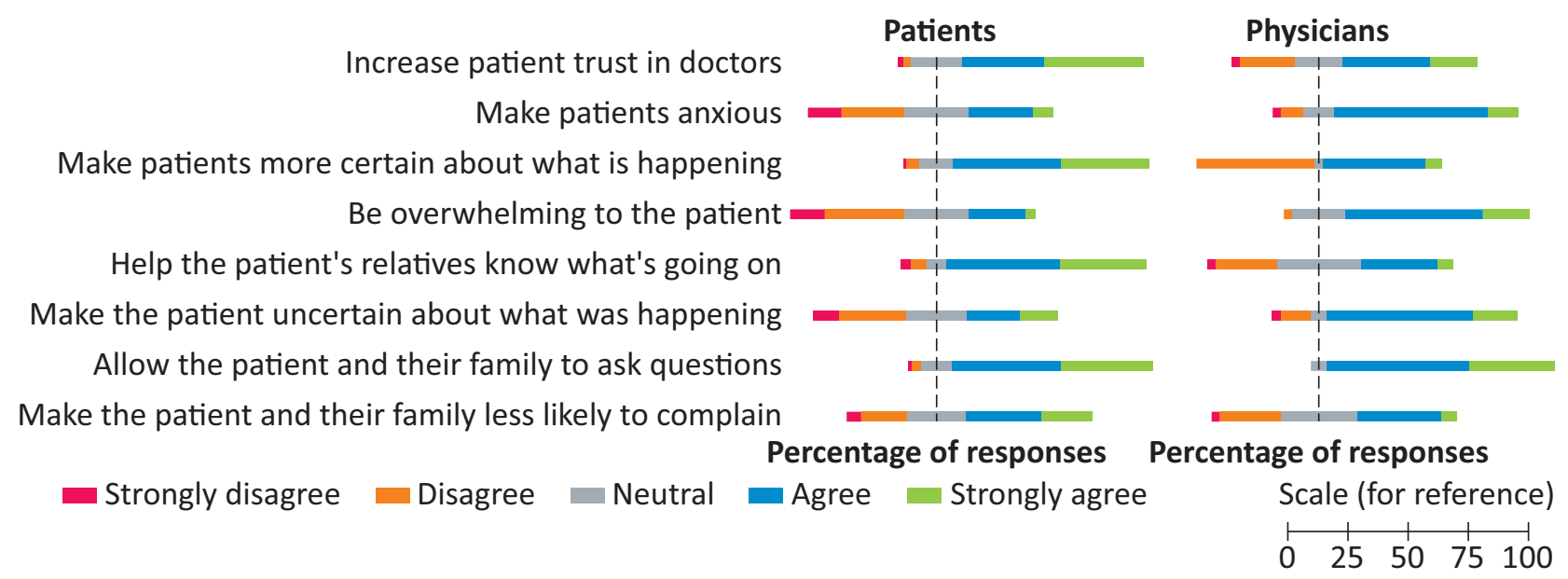

Fig 2. Patient and physician responses to the prompt, 'Having access to medical records would...'. Physicians were asked to respond based on how they felt this would affect patients. The five-point Likert data are plotted as a diverging stacked bar graph; each bar equates to $100 \%$ of the response to that question, centred about the neutral responses.

thought it would create more uncertainty $(\mathrm{m}=3.98)$. Physicians thought that sharing the medical record would make patients anxious $(m=3.91)$, and potentially overwhelm them $(m=3.95)$; patients were less likely to share these concerns (anxiety $m=2.92$; overwhelming $\mathrm{m}=2.64$; see Fig 2).

Most physicians (81\%) thought that sharing the medical record with patients during their stay would change what they write in the record.

Free text answers provided a range of reasons for and against realtime sharing of the medical record (see Table 1). Patients thought they had a right to information about themselves, that sharing would help their knowledge and recall of events, but that medical terminology might be confusing. Physicians thought sharing might improve partnership with patients and encourage questions but might create anxiety and change what is documented.

Patients and physicians favoured a summary record although physicians were less supportive ( $m=3.67$ for physicians vs 4.07 for patients). Patients thought the summary record might be easier to understand, and less overwhelming. Physicians thought it might act as a platform for discussion. Details of what information would be preferred in the summary by both patients and physicians can be found in Supplement S4. Both were concerned about the impact on workload.

Just over half of patients ( $56 \%$ ) preferred access to a summary record to access to the full record.

\section{Information sharing, uncertainty and trust}

Physicians were asked about their current practice. All participants were asked for their views on how certain practices, used during information-sharing, affected patient trust (see Fig 3).

Most physicians reported communicating uncertainty in diagnosis (79\%; $m=3.98)$. Physicians thought it would increase trust $(m=3.95)$ whereas patients thought it would decrease it $(m=2.87)$.

Physicians varied in their practice of telling patients about mildly deranged results $(m=3.46)$ or investigations to exclude sinister causes of their symptoms $(m=2.95)$. Arguments for sharing such information included transparency and involving the patient in decision making; this was balanced against causing 'unnecessary anxiety'.

\section{Discussion}

This study found that with current practices for sharing information, $27 \%$ of patients did not know their diagnosis immediately after a hospital stay, and 39\% did not know what treatments they had been given at the time of questionnaire completion.

Despite reporting feeling able to ask questions, most patients did not do so.

There were discrepancies between physician and patient attitudes towards sharing the medical record in real time, in particular in relation to the effects that sharing the record might have on anxiety, trust and what was written in the record. Both groups supported sharing a summary record, although there were concerns about resource implications.

It is possible that lack of reported knowledge from the patients reflects lack of recall rather than failure of information provision; in a study of healthy volunteers, only $25 \%$ were able to spontaneously recall the information about an anaesthetic they were given. ${ }^{18}$ It has previously been noted that patients' normal level of questioning can be affected by the presence of a physician, so-called 'white coat silence,. ${ }^{19}$ The lack of reported questioning in this study may reflect that patients felt they have sufficient information, did not recall sufficient information on which to base questions or were demonstrating 'white coat silence'.

The discrepancy between patients' and physicians' views on whether the full medical record should be shared in real time was notable. Previous studies in the outpatient ${ }^{20}$ and emergency settings ${ }^{21}$ were consistent with ours in suggesting that physicians were concerned about causing anxiety, overwhelming patients, generating questions and increasing uncertainty, while patients were not. This may be because most patients are unaware of exactly what is included in the patient record (for example differential diagnoses and mildly abnormal investigation results would not normally be shared with patients unless they became relevant to the patients' care) and therefore unaware of potential anxiety-provoking data. Whether physicians or patients are correct about the risk of access to records creating undue anxiety 


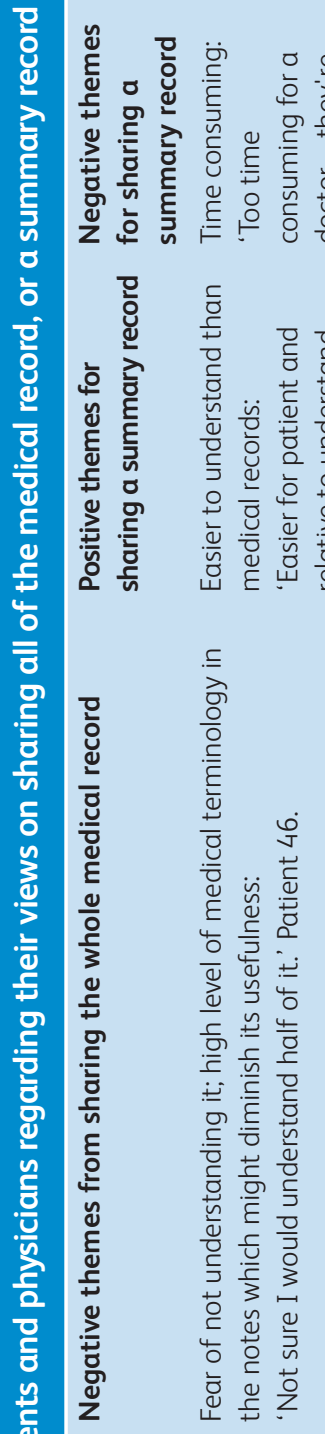

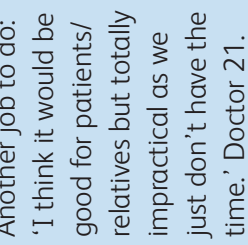

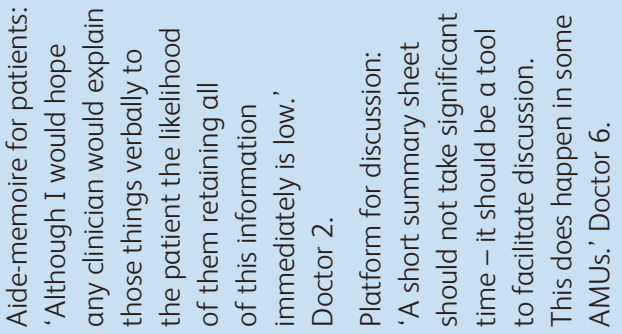

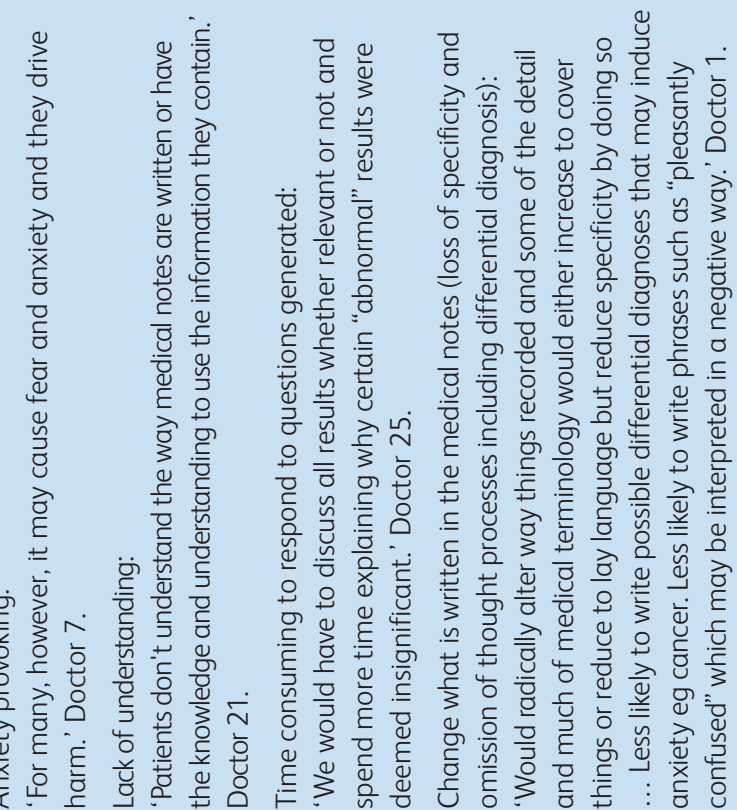

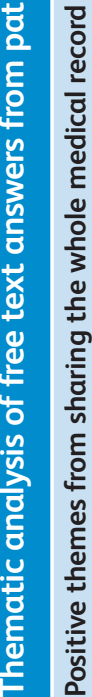

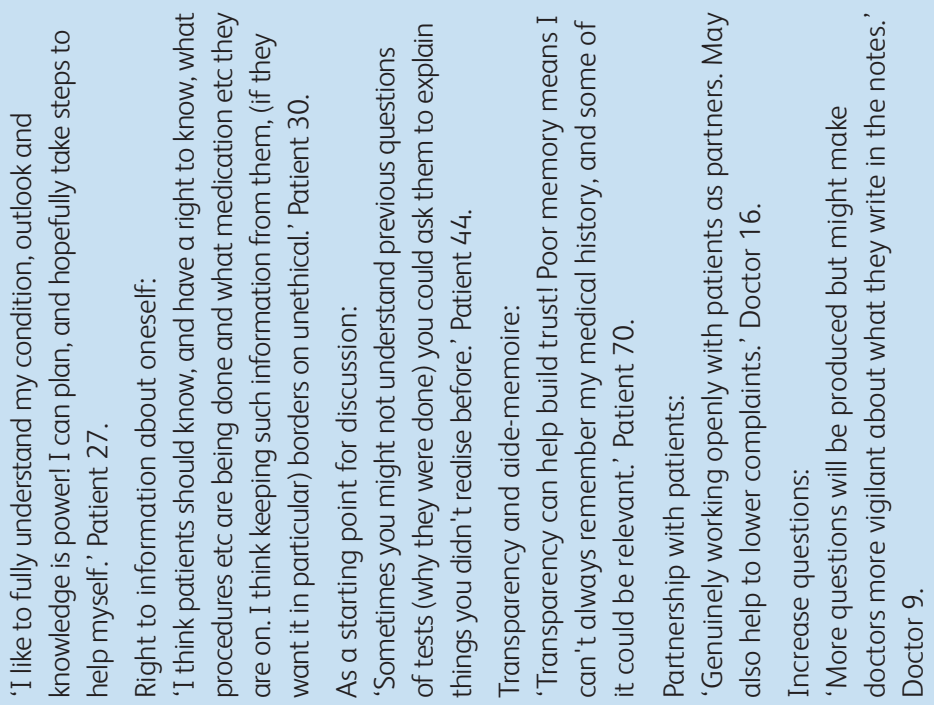


Suggested you write down questions

Showed you your medical notes

Talked about their own experiences

Talked about things unrelated to your medical condition

\section{Expressed confidence \\ Explained uncertainty in diagnosis
Decreased trust a lot Decreased trust a little Increases trust a little Increases trust a lot

Percentage of responses

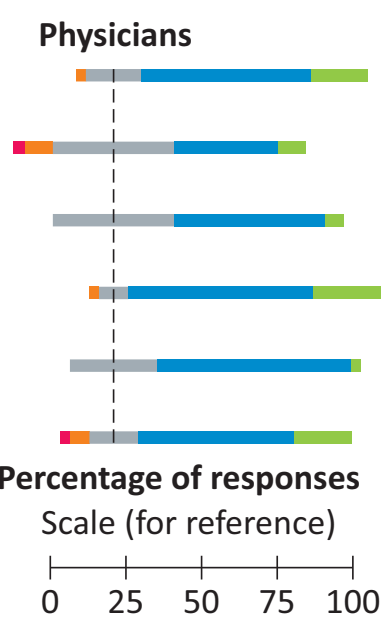

Fig 3. Patient and physician responses to how they felt trust was affected when physicians did certain things (listed on y axis). Physicians were asked to respond based on how they felt patient trust in them would be affected. The five-point Likert data are plotted as a diverging stacked bar graph; each bar equates to $100 \%$ of the response to that question, centred about the neutral responses.

can be tested empirically through intervention studies of record sharing. Studies in outpatient settings have shown that physicians' concerns about increased patient anxiety and visit requests were unfounded. ${ }^{8,22}$ Cancer patients who were given full access to their notes did not have increased anxiety, ${ }^{23}$ no similar studies in the acute setting have yet been conducted.

Physicians questioned in this study believed that sharing diagnostic uncertainty would increase trust, whereas patients responded that they thought it would decrease it. The physicians' view may be informed by moral discomfort about the routine non-disclosure which was reported by them in this study. No other corroborative or contradictory studies on routine non-disclosure could be found, although the moral equivalence of non-disclosure and lying in medicine has been explored. ${ }^{24}$ Patients' perception that physicians sharing uncertainty might invoke less trust might reflect a belief that uncertainty represents incompetence, rather than being commonplace. This needs to be better understood, so that we can, as Simpkin has stated, better train physicians in how to tolerate and communicate uncertainty. ${ }^{25}$

The majority of physicians expressed concern that sharing the medical record will change what is recorded. Previous studies on record sharing have not considered this issue. Possible reasons for physician concern could include the risk of investigations to exclude unlikely but important diagnoses not being done, or risk of over investigation of some patients because of a reticence to document a concern about somatisation disorder. However, sharing the medical record may result in a positive change in recording with less use of jargon and clearer justification of decisions. It seems likely that sharing the record will lead to some change in documentation and future studies should specifically investigate this.

\section{A summary record: a balance between transparency and information-overload?}

Arguments in favour of sharing the record with the patient have been made based on the patients' right to know information about themselves, ${ }^{26}$ and the empowerment that access induces. ${ }^{27}$
Such empowerment could be offered by a summary record without causing the potential adverse effects that physicians are concerned might happen with access to the whole record. A summary record was the favoured approach expressed by the patient and physician participants in this study, although there were concerns about the time it might take. One approach to a summary record which has been evaluated is the "patient portal'. ${ }^{28}$ A review of six adopters suggested that access to problem lists and care goals would be valuable. ${ }^{29}$ However, the only controlled trial conducted did not demonstrate any improvement in patient knowledge with access to the portal. ${ }^{30}$

\section{Strengths and limitations}

This is the first UK study investigating patient and physician attitudes to having access to medical records in the inpatient setting. We achieved a high response rate in a large number of patients reporting their recent experience. Physicians and patients provided extensive free text responses, adding richness to the data. The low number of physicians completing the questionnaire meant that only descriptive statistical comparisons could be made between patient and physician responses, and no subgroup analyses were possible. Some patients were aided by nursing staff in completing the questionnaire (the nurse read the questions and documented the answers) and it is possible that this affected the responses.

Patients who lacked capacity or were unable to read English were not eligible for participation, we were therefore unable to explore the experiences and views of these patients.

\section{Conclusion}

Many patients conclude their admission for an acute illness without knowing their diagnosis or treatment, and few patients appear to question their physicians about their care. This suggests a failure in the current system of providing information verbally to patients in this setting. Patients and physicians support the proposal to share the written medical record (or a summary of this) 
with the patient but have different views about the consequences of disclosing more information including its effect on patient anxiety and trust.

Any change to sharing either a summary or full medical record should be evaluated to identify the impact on professional practices and on patient experience and care.

\section{Acknowledgments}

We would like to thank Prof Frances Griffiths for her very helpful contribution to the development of the questionnaire; Sarah Bunnewell, Hanna Alton-Reynolds, Emily Chruscikowski and Daniel Newport for contributing to data inputting; research nurses Linda Webber, Faye Moore, Cheryl Davis, Ann-Marie O'Sullivan and Alice Robertson for their outstanding work recruiting and distributing the patient questionnaire; and finally the doctors and patients who took the time to complete the questionnaire.

\section{Supplementary material}

Additional supplementary material may be found in the online version of this article at http://www.clinmed.rcpjournal.org:

S1 - Questionnaires.

S2 - Demographic data.

S3 - Patient information.

S4-Preferred information.

\section{References}

1 Delbanco T, Walker ], Bell SK et al. Inviting patients to read their physicians' notes: a quasi-experimental study and a look ahead. Ann Intern Med 2012;157:461-70.

2 Ayana M, Pound P, Lampe F et al. Improving stroke patients' care: a patient held record is not enough. BMC Health Serv Res 2001;1:1.

3 Elbourne D, Richardson M, Chalmers I et al. The Newbury Maternity Care Study: a randomized controlled trial to assess a policy of women holding their own obstetric records. $\mathrm{Br}$ J Obstet Gynaecol 1987;94:612-9.

4 Walker J, Leveille SG, Ngo L et al. Inviting patients to read their physicians' notes: patients and physicians look ahead: patient and physician surveys. Ann Intern Med 2011;155:811-9.

5 Edgman-Levitan S, Cleary PD. What information do consumers want and need? Health Aff (Millwood) 1996;15:42-56.

6 Delbanco T, Berwick DM, Boufford JI et al. Healthcare in a land called PeoplePower: nothing about me without me. Health Expect 2001;4:144-50.

7 Coulter A. Engaging patients in their healthcare: How is the UK doing relative to other countries? Oxford: Picker Institute Europe, 2006.

8 Bell SK, Mejilla R, Anselmo M et al. When physicians share visit notes with patients: a study of patient and physician perceptions of documentation errors, safety opportunities and the patientphysician relationship. BMJ Qual Saf 2017;26:262-70.

9 Leveille SG, Mejilla R, Long N et al. Do patients who access clinical information on patient internet portals have more primary care visits? Medical Care 2016;54:17-23.

10 Kelly MM, Coller RJ, Hoonakker PL. Inpatient portals for hospitalized patients and caregivers: a systematic review. J Hosp Med 2018;13:405-12.
11 Woollen J, Prey ], Wilcox L et al. Patient experiences using an inpatient personal health record. Appl Clin Inform 2016;7:446-60.

12 O'Leary KJ, Sharma RK, Killarney A et al. Patients' and healthcare providers' perceptions of a mobile portal application for hospitalized patients. BMC Med Inform Decis Mak 2016;16:123.

13 Pell JM, Mancuso M, Limon S et al. Patient access to electronic health records during hospitalization. JAMA Intern Med 2015;175:856-8.

14 Access to Health Records Act 1990. London: Her Majesty's Stationery Office, 1990.

15 Weinert C. Giving physicians' daily progress notes to hospitalized patients and families to improve patient experience. Am J Med Qual 2017:32:58-65.

16 Jobe JB, Mingay DJ. Cognitive research improves questionnaires. Am J Public Health 1989;79:1053-5.

17 Sullivan GM, Artino ARJr. Analyzing and interpreting data from likert-type scales. J Grad Med Educ 2013;5:541-2.

18 Sandberg EH, Sharma R, Sandberg WS. Deficits in retention for verbally presented medical information. Anesthesiology 2012;117:772-9.

19 Judson TJ, Detsky AS, Press MJ. Encouraging patients to ask questions: how to overcome 'white-coat silence'. JAMA 2013;309:2325-6.

20 Delbanco T, Walker J, Darer JD et al. Open notes: physicians and patients signing on. Ann Intern Med 2010;153:121-5.

21 Wilcox LG, Gatewood J, Morris D et al. Physician attitudes about patient-facing information displays at an urban emergency department. AMIA Annu Symp Proc 2010;2010:887-91.

22 Mishra VK, Hoyt RE, Wolver SE et al. Qualitative and quantitative analysis of patients' perceptions of the patient portal experience with OpenNotes. Appl Clin Inform 2019;10:10-8.

23 Gravis $G$, Protière $C$, Eisinger $F$ et al. Full access to medical records does not modify anxiety in cancer patients: Results of a randomized study. Cancer 2011;117:4796-804.

24 Cox CL, Fritz Z. Should non-disclosures be considered as morally equivalent to lies within the physician-patient relationship? J Med Ethics 2016;42:632-5.

25 Simpkin AL, Schwartzstein RM. Tolerating uncertainty - the next medical revolution? N Engl J Med 2016;375:1713-5.

26 Gilhooly ML, McGhee SM. Medical records: practicalities and principles of patient possession. J Med Ethics 1991;17:138-43.

27 Wibe T, Helleso R, Slaughter L et al. Lay people's experiences with reading their medical record. Soc Sci Med 2011;72:1570-3.

28 Dykes PC, Carroll DL, Hurley AC et al. Building and testing a patientcentric electronic bedside communication center. J Gerontol Nurs 2013;39:15-9.

29 Grossman LV, Choi SW, Collins S et al. Implementation of acute care patient portals: recommendations on utility and use from six early adopters. J Am Med Inform Assoc 2018;25:370-9.

30 O'Leary KJ, Lohman ME, Culver E et al. The effect of tablet computers with a mobile patient portal application on hospitalized patients' knowledge and activation. J Am Med Inform Assoc 2016;23:159-65.

Address for correspondence: Dr Zoë Fritz, The Healthcare Improvement Studies (THIS) Institute, University of Cambridge, Clifford Allbutt Building Cambridge Biomedical Campus, Cambridge CB2 OAH, UK.

Email: zoe.fritz@addenbrookes.nhs.uk 